\title{
Successful Mobile Application Development: Towards a Taxonomy of Domain-Specific Process Models and Methodologies
}

\author{
Oliver Werth \\ Leibniz Universität Hannover \\ werth@iwi.uni-hannover.de
}

\author{
Nadine Guhr \\ Leibniz Universität Hannover \\ guhr@iwi.uni-hannover.de
}

\author{
Michael H. Breitner \\ Leibniz Universität Hannover \\ breitner@iwi.uni-hannover.de
}

\begin{abstract}
Mobile applications and mobile application development issues receive an increasing attention for practitioners and academics. The development of mobile applications is connected with a number of domain-specific issues and challenges (e.g., fulfilment of customer requirements or the prevention of high development costs). Consequently, the decision of the most effective process model to develop a mobile application plays a crucial role for software and mobile application development teams. With the help of a structured taxonomy-building methodology, we contribute to the extant literature by creating and presenting a taxonomy for process models and methodologies in software engineering and the mobile application development domain. The taxonomy enrich the existing knowledge base and can help mobile application developers to choose the most suitable process model or methodology. Based on our examination, our results indicate new directions for mobile application research and implications for mobile application development.
\end{abstract}

\section{Introduction}

In the last ten years, mobile applications have received an ongoing interest in both the private and the professional area, resulting in an exponential growth in mobile application development [1]. Until 2018, the number of mobile applications has raised to nearly four million applications in Google PlayStore or two million in Apple's App Store [2]. Because of this raising number and huge supply of mobile applications, the market had become very competitive because since users can switch easily to another application [3]. Therefore, developers have to consider and ultimately meet customer requirements (e.g., usability [4] or improve the experience [5]) adequate and quick [6].
As Majchrzak et al. [7; p. 5735] already stated "the basic requirements of developing mobile applications can be said to have become less complex and more complex at the same time". Various customer requirements are followed by a raising number of special technical issues for mobile applications and should therefore be taken into account in the development process.

Methods from classical software development like iterative, parallel or sequential process models can be used by some extent, but new processes are necessary because of the distinctive nature and the characteristics of mobile applications, e.g., characteristics related to hardware (e.g., compatibility, performance restrictions, battery life), characteristics related to software (e.g., integration, interaction, error notification, convenience, reachability), and characteristics related to communication (e.g., network connectivity) $[8,9,10]$. The differentiation of process models into a few generic archetypes leads to a sometimes meaningful reduction in complexity, we argue that for some purposes, such as the development of a mobile application, a more detailed domainspecific perspective is necessary. This increasing importance and complexity makes it necessary to implement more flexible and specialized procedures that meet the specific needs regarding the development of mobile applications.

To our understanding, there is limited academic literature that incorporates and investigates process models and methodologies into the mobile application domain. This pressing problem for practitioners is reflected in research, where studies have emphasized that the selection of an appropriate process model for the development of a mobile application represent a current dilemma in theory and practice [11]. However, the role of process models and methodologies in the context of mobile application development has been considered in only a few research studies. Therefore, the aim of this research is to organize and structure the amount of knowledge from academic literature in order to create and present a taxonomy of process 
models and methodologies in software engineering and the mobile application development domain. We will demonstrate how the process model concept helps to improve the understanding of the mobile application development process.

Kemper \& Wolf [11; p. 409] stated that in practice, process models (e.g., incremental models) often resemble a "quick-and-dirty" procedure, which are not sufficient for the development of mobile applications. They argue that it is necessary to synthesize existing development processes for mobile application as a basis for the optimizing and conceptualizing of process models in order to meet the challenges associated with a successful mobile application development.

Therefore, we structure existing process models and methodologies by means of a taxonomic approach following Nickerson et al. [12]. Such classification has many benefits, e.g., understanding and analyzing research areas, and creating a common understanding and terminology in research [13]. We deliberately chose a taxonomy, which plays a significant role in research and practice, because a taxonomy has the ability to structure concepts and relationships and show differences in research results $[14,15]$. To address the identified research gap, we address the following research question:

$R Q:$ What process models and methodologies can be applied in the mobile application development domain and how can they be classified?

The remainder of this paper is organized as follows. In the first section, we give a theoretical background on the different characterizations of mobile applications and development issues and give an understanding on process models and methodologies especially in software engineering. Second, we describe our methodology of taxonomy building according to Nickerson et al. [12]. After this, the created taxonomy is derived in section four. Following this, the identified characteristics and dimension are discussed. We critically reflect and discuss our research results and contributions into the mobile application research domain and give theoretical and practical implications for academics and mobile application developers. Before we conclude with a brief summary, we point out limitations and give an outlook for future research.

\section{Theoretical background}

\subsection{Characterizations of mobile applications and application development issues}

In order to receive a consistent understanding of what we mean by a mobile application we will first give a working definition for our research. Mobile applications can be defined as "an (information technology) IT software artifact that is specifically developed for mobile operating systems installed on handheld devices, such as smartphones or tablet computers" [4; p. 437). Numerous mobile applications have been examined in literature. Nickerson et al. [12] for example, examined mobile applications like mobile messaging or mobile games. Han et al. [16] investigated mobile application analytics more in detail and presented different application categories, like communication or entertainment, based on a longitudinal study of panel data.

Nickerson et al. [12] constructed a taxonomy for mobile applications and examined different characteristics: (1) temporal (user interaction with application happens synchronously or asynchronously), (2) communication (information can flow from the application to the user, vice versa or in both ways), (3) transaction (the user makes a financial transaction through the application or not), (4) access (application can only use by restricted users or can be used by anyone), (5) multiplicity (application has a single or multiple user), (6) location (application uses the location of the user or not), (7) identity (application uses the identity of the user or not).

Another examination of mobile application characteristics is from Flora et al. [8]. Using a mixedmethods approach, they interviewed several mobile application developers worldwide about specific characteristics of mobile applications. In result, they examined three sets of different characteristics related to the hardware (e.g., screen size), to the software (e.g., error notification) and to the communication (e.g., network connectivity) of mobile applications [8].

In addition to the number of diverse characteristics of mobile applications from Flora et al. [10] and Majchrzak \& Grønli [8], several domain-specific software development issues, which are, of course, connected to the characteristics, have to be also incorporated into the development process. These challenges have been widely examined in the scientific literature, which we shortly outline (e.g., $[1,5,17,18,19])$.

Developers of mobile applications should be aware of the communication of the planned application with other applications, because of e.g., security issues. Other issues are the inclusion and usage of sensors of mobile devices, the different behaviors of native or hybrid applications, and the different families of hardand software (e.g., the operation system on the device). In addition, security issues, e.g., for the personalized data of the user, the specific user 
interface (UI), the testing complexity of the planned application, and the power consumption of the application from the mobile device should be taken into consideration [1].

Dehlinger \& Dixon [17], as another example, specified on central issues, like the design of contextawareness of the application or the balance between agility and requirements of the mobile application.

Joorabchi et al. [18] were able to identify four grand challenges in their mixed-methods research approach. Participants in their study acknowledged the existence of general challenges (e.g., data intensity of the applications), challenges in multi-platform development as well as challenges in testing and analyzing the application [18].

A study of the mobile learning context showed specific issues such as data sharing and collaboration features needed to be critically determined within the development process [19].

In a mobile application development process it is important to know both the category and the characteristics of the intended application. It is shown that mobile applications can be classified into multiple domain-specific dimensions, characteristics, and development issues that must be considered by everyone involved in the development process. We consider this diversity in our taxonomy building procedure as well as for our discussion and reflection of our study.

\subsection{Processes and process models in software engineering}

Focusing on mobile applications and their varying characteristics as a software artifact, we give an understanding about processes and process models in the software engineering domain. According to Pressmann \& Maxim [20], software development processes can be generally arranged into five main activities: (1) communication (contains conversation with the stakeholders about the requirements of the software and documentation), (2) planning (means to use of a set of management and technical practices to define a structured plan to achieve the software), (3) modeling (helps the developer to catch the software from a customer's perspective by modeling customer requirements and/or the architecture or user interface of the planned software), (4) construction (contains all coding and testing tasks to build a software which ca be delivered to the stakeholder), and (5) deployment (contains delivery of software to the customer, provides support for the customer and contains feedback for the software development team for further development). The specificity and execution of these processes (stages) differs significantly from process model to process model, which we describe later in our taxonomic approach.

With regard to process models, they can be defined as a sequence of events leading to an outcome [21]. They have several goals for organizations. For example, process models should help the members of an organization to automate and integrate business processes within the organization. In addition, they should help workers to analyze and rearrange their activities $[22,23,24]$. When we look at process models in the software development domain, they "represent a networked sequence of activities, objects, transformations, and events that embody strategies for accomplishing software" [25; p. 4]. Process models in software development can be classified into nonoperational and operational types. Operational models are scripts and programs that help the developer to develop the software in their process. Non-operational models, on the other hand are not automated and are more conceptual models (e.g., the spiral model of software development by Boehm [26]. In our approach we focus on non-automated process models.

The development of mobile application as a software is connected with a number of different tasks and procedures and involves a number of persons [27]. Therefore, practitioners and academics use and adapt traditional software development process models in order to structure their development process. We will structure these traditional and adapted software development process models and methodologies and connect them with our understanding of specific mobile application development issues.

\section{Methodology}

As mentioned by Nickerson et al. [12; p.1] "a fundamental problem in many disciplines is the classification of objects of interest into taxonomies". However, taxonomies play an important role in research not only in social science research or biology but also in information systems research in order to analyze complex domains, to provide an organization, and structure to the knowledge of a field and to gain a deeper understanding of new objects $[12,28,14]$. Such a classification can be used to understand and pursue research and allows to grasp and analyze complex issues [29]. The methodology of Nickerson et al. [12] has been adapted in several research areas. Prat et al. [30] used this taxonomic approach in order to build a taxonomy for evaluation methods of information systems artifacts in the design science research area [31]. Another example is the study of Remane et al. [28]. The authors used this approach to structure 
carsharing business models. Most recent, Gimpel et al. [32], constructed a taxonomy of service offerings in the FinTech sector. Although there is some discussion regarding the conceptual use of typology, framework, and taxonomy, we will not go into more detail here because of the limited space available. In our paper, we use the term taxonomy as an approach to arrange and characterize different process models and methodologies into ideally homogenous groups. Our research approach to creating a taxonomy of domainspecific process models and methodologies for mobile applications is based on the design science paradigm oriented iterative taxonomy development method by Nickerson et al. [12]. The taxonomy development process consists of seven steps (see Fig. 1).

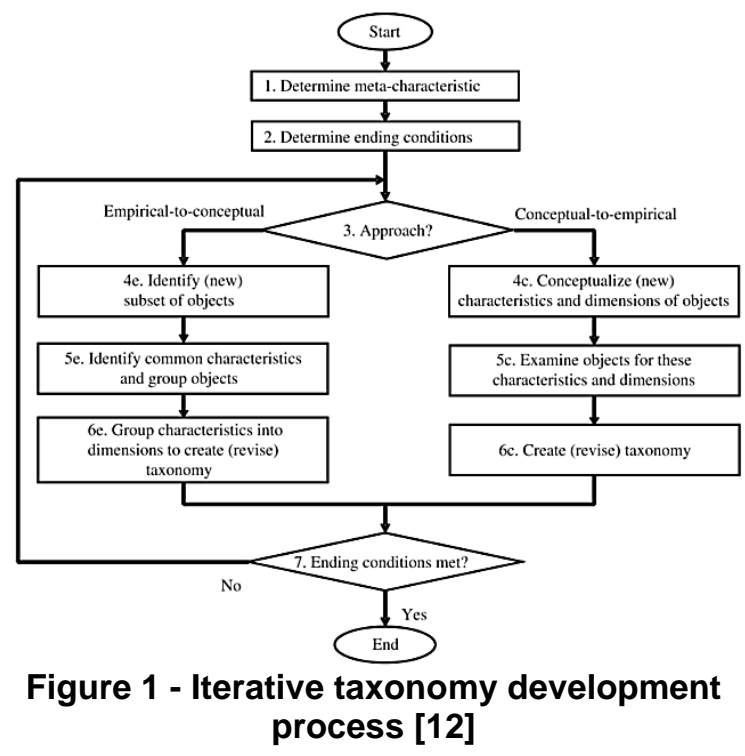

First, as a basis, the meta-characteristics, which reflect the purpose of the taxonomy, must be specified [12]. These characteristics serve as foundation for all other characteristics of the taxonomy so that all characteristics of the taxonomy are a logical consequence of the meta-characteristic and aid in describing the structural differences of process models and methodologies for mobile applications. As the main goal is to characterize process models and more specific process models in mobile application development, we offer high level characteristics, which are especially interesting for researchers and developers of mobile applications.

We identified the meta-characteristic for the process models based on a detailed literature search. Due to the multitude of process models in different research domains, we could not identify a process model, which serves as a framework. As metacharacteristic, we chose the design of the process to develop a software or mobile application (e.g., a communication phase or a planning phase described in the aforementioned theoretical background).

Second, after the meta-characteristics are outlined, the ending conditions to terminate the process should be determined. According to Nickerson et al. [12] we adopted three objective ending conditions ("No new dimensions or characteristics were added in the last iteration", "No dimensions or characteristics were merged or split in the last iteration", and "Every characteristic is unique within its dimension") and five subjective ending conditions - concise, robust, comprehensive, extendible, and explanatory - as qualitative attributes for our taxonomy. By observing the necessary subjective ending conditions, it is ensured that the taxonomy contains a limited number of dimensions and characteristics in each dimension. The taxonomy must contain enough dimensions and characteristics to allow a clear differentiation of the objects of interest. It must classify all known objects within the respective domain and must also include all dimensions of the objects that are of interest. By using a taxonomy it must be possible to include additional dimensions and characteristics if new forms of objects appear and to identify where an object is found in the taxonomy respectively the characteristics of an object is found in the taxonomy [12,29].

Third, the method allows two distinct cycles conceptual-to-empirical and empirical-to-conceptual - in iterative passes. The choice of an approach depends largely on the researchers' understanding of the domain and the availability of data about the objects [33]. The empirical-to-conceptual (inductive) approach means that a subset of the objects to be classified must be evaluated for common characteristics and dimensions, which are then added to the taxonomy. The other approach is conceptual-toempirical (deductive), which means that the dimensions and characteristics may be derived from the literature and the knowledge of the authors. During our research process and the different iterations (four iterations), we used both approaches. We decided to use the conceptual-to-empirical approach when we thought that we could conceive and refine additional dimensions and we adapted the empirical-toconceptual approach when we thought that there are more process models or methodologies to examine. In two iterations ( 1 and 2 ) we used the empirical-toconceptual approach and in iteration 3, we used the conceptual-to-empirical approach to derive a diverse set of dimensions and characteristics. Another empirical-to-conceptual iteration (iteration 4) concludes our taxonomy. 


\section{Towards a taxonomy of domain-specific process models and methodologies}

We present our taxonomy based on the methodology described in the section before. On the one hand, this taxonomy provides us an overview of different process models and methodologies as well as similar or different dimensions. On the other hand, it provides us a basis for our analysis in order to make contributions to the mobile application development area. As a remainder, the meta-characteristic of our taxonomy is the development of a mobile application. Therefore, we are looking for process models and methodologies in software engineering and mobile application development.

In the first iteration, we identified the following process models: Waterfall model (WM) [34], spiral model (SM) [26], V-model (VM) [35], incremental development model (IDM) [36], concurrent development model (CDM) [20], component-based software development model (CSDM) [20], extreme programming (XP) [37], SCRUM [38], dynamic systems development method (DSDM) [39], feature driven development (FDD) [40], and the rational unified process (RUP) [41]. These process models were included through own knowledge and experiences in (mobile) software development and engineering of the authors.

After identifying a first set of process models and methodologies, we compared them for differences and similarities and assigned these process models and methodologies to the first identified dimensions. Our first dimension, was "phases". Three characteristics were identified, namely sequential, iterative, and parallel. Some process models follow a linear sequence of tasks, while others follow a parallel or iterative approach. In addition, we found differences in the process models in terms of development efforts. These efforts are described sometimes as "low" (e.g., at the CSCM) and sometimes as "high" in order to develop the software. For example, CDM [20] demands only for a limited ability to construct and program an application. This results from the fact that the development process is divided into several parallel processes, with some parallel sub-processes in a waiting phase, while other activities or sub-processes (such as programming) can be fulfilled. In result, the effort is lower than in other models, because the development team can focus on one activity. Our third dimension focuses on the knowledge of customer requirements, which plays an important role in software engineering [20]. We defined this knowledge as partially-known and well-known in the beginning of the software development process. Various process models, such as the WM, require complete information regarding the software developed. Other process models (e.g., SCRUM) do not need the full information at the beginning or incorporates the requirements to the software in the development process. Prototyping was identified in process models also as a crucial dimension, which is necessary to mention and, therefore, to include in our taxonomy. Prototyping, in general, is a crucial process in software development and helps to meet the customer requirement more effective [42]. We found out that prototyping is incremental in most process models, like in XP. Incremental means that, prototyping takes place at least once in the software development process. Other models, do not include prototyping at all (e.g., the WM). Our fifth dimension identified dimension is "involvement of the costumer", which we characterized as "singular" (customer is involved only one-time in the development process) and "often" (customer is involved multiple times into the development process) in our first iteration. This dimension describes the number of involvements of the customer inside the entire software development process.

Our second iteration incorporates process models and modifications of them, which are used in a mobile application domain-specific context. Exploratory key word searches in (meta-) databases such as the AIS eLibrary, EBSCOhost or ScienceDirect, which includes software engineering and computer sciencespecific journals and conference proceedings, guides our search. Keywords and keyword strings like "process model", "process model for mobile application" or "mobile application development" supported our search procedures. In order to receive a more comprehensive view, we do not restrict in terms of publication date or journal rankings. We examined three additional objects (process models and methodologies) for our taxonomy. We added MobileD [43], the iterative process models for mobile applications (IPM) [11] and lean software development (LSD) [44]. We classified these new objects into our existing dimensions and characteristics from the first iteration.

In our third iteration (conceptual-to-empirical), we have not added any new objects. Rather, we believe that we should expand and refine the dimensions, as existing ones because the existing ones do not properly explain the characteristics of the methodologies. Therefore, we expanded "involvement of the customer" with the characteristic of "permanent" (customer is involved all the time in the development process). In order to achieve a better delimitation of the characteristics, we renamed "often" to "regular". 
As we read more literature and articles in journals and conference proceedings through forward and backward search [45], we decided to conduct another iteration because we felt we could find even more process models and methodologies. In this fourth iteration (empirical-to-conceptual) we found two additional objects for our taxonomy, which we incorporated. Namely the MASAM methodology [46] and the perspective-dissolve approach for mobile application development (MobiPDA) [47]. After this forth iteration, we have not found further process models and methodologies in software engineering and mobile application development. In addition, all included process models and methodologies were classified and three objective and five subjective ending conditions are satisfied, thereby ending the iterations. Table 1 shows our developed taxonomy for process models and methodologies and their associated dimensions and characteristics. In total, we examined 16 objects (process models and methodologies), five dimensions and 12 characteristics.

Table 1 - Taxonomy for process models and methodologies after four iterations

\begin{tabular}{|c|c|c|c|c|c|c|c|c|c|c|c|c|}
\hline \multirow{4}{*}{ Objects } & \multicolumn{12}{|c|}{ Core Taxonomy Dimensions } \\
\hline & \multicolumn{3}{|c|}{ Phases } & \multicolumn{2}{|c|}{$\begin{array}{c}\text { Develop- } \\
\text { ment efforts }\end{array}$} & \multicolumn{2}{|c|}{$\begin{array}{c}\text { Requirements of } \\
\text { customers }\end{array}$} & \multicolumn{2}{|c|}{ Prototyping } & \multicolumn{3}{|c|}{ Involvement of the customer } \\
\hline & \multicolumn{12}{|c|}{ Characteristics } \\
\hline & Sequential & Iterative & Parallel & Low & High & $\begin{array}{c}\text { Partially- } \\
\text { known }\end{array}$ & $\begin{array}{c}\text { Well- } \\
\text { known }\end{array}$ & None & Incremental & Singular & Regular & $\begin{array}{c}\text { Per- } \\
\text { manent }\end{array}$ \\
\hline WM [34] & $\mathrm{x}$ & & & $\mathrm{x}$ & & & $\mathrm{x}$ & $\mathrm{x}$ & & $\mathrm{x}$ & & \\
\hline SM [26] & & $\mathrm{x}$ & & & $\mathrm{x}$ & $\mathrm{x}$ & & & $\mathrm{x}$ & & $\mathrm{x}$ & \\
\hline VM [35] & & $\mathrm{x}$ & & & $\mathrm{x}$ & $\mathrm{x}$ & & $\mathrm{x}$ & & & $\mathrm{x}$ & \\
\hline IDM [36] & & & $\mathrm{x}$ & & $\mathrm{x}$ & $\mathrm{x}$ & & & $\mathrm{x}$ & & $\mathrm{x}$ & \\
\hline CDM [20] & & & $\mathrm{x}$ & $\mathrm{x}$ & & $\mathrm{x}$ & & & $\mathrm{x}$ & & $\mathrm{x}$ & \\
\hline CSDM [20] & & $\mathrm{x}$ & & $\mathrm{x}$ & & & $\mathrm{x}$ & & $\mathrm{x}$ & $\mathrm{x}$ & & \\
\hline $\mathrm{XP}[37]$ & & $\mathrm{x}$ & & $\mathrm{x}$ & & $\mathrm{x}$ & & & $\mathrm{x}$ & & & $\mathrm{x}$ \\
\hline SCRUM [38] & & $\mathrm{x}$ & & & $\mathrm{x}$ & & $\mathrm{x}$ & & $\mathrm{x}$ & & $\mathrm{x}$ & \\
\hline DSDM [39] & & $\mathrm{x}$ & & $\mathrm{x}$ & & $\mathrm{x}$ & & & $\mathrm{x}$ & & $\mathrm{x}$ & \\
\hline FDD [40] & $\mathrm{x}$ & & & & $\mathrm{x}$ & & $\mathrm{x}$ & & $\mathrm{x}$ & & & $\mathrm{x}$ \\
\hline RUP [41] & & $\mathrm{x}$ & & & $\mathrm{x}$ & $\mathrm{x}$ & & & $\mathrm{x}$ & & & $\mathrm{x}$ \\
\hline Mobile-D [43] & & $\mathrm{x}$ & & & $\mathrm{x}$ & $\mathrm{x}$ & & & $\mathrm{x}$ & & $\mathrm{x}$ & \\
\hline IPM [8] & & $\mathrm{x}$ & & & $\mathrm{x}$ & $\mathrm{x}$ & & & $\mathrm{x}$ & & $\mathrm{x}$ & \\
\hline LSD [44] & & $\mathrm{x}$ & & & $\mathrm{x}$ & $\mathrm{x}$ & & & $\mathrm{x}$ & & & $\mathrm{x}$ \\
\hline MASAM [46] & & $\mathrm{x}$ & & & $\mathrm{x}$ & $\mathrm{x}$ & & & $\mathrm{x}$ & & & $\mathrm{x}$ \\
\hline MobiPDA [47] & & $\mathrm{x}$ & & $\mathrm{x}$ & & & $\mathrm{x}$ & & $\mathrm{x}$ & $\mathrm{x}$ & & \\
\hline
\end{tabular}

Several observations can be made. Regarding the dimension of "phases", we found that most of the examined process models and methodologies follow an iterative process flow in order to develop the software. For example, WM and FDD contain sequential phases in software development while, on the other hand, IDM and CDM are follow a parallel approach in order to develop the software.

The development efforts, mentioned in the description of the models in order to further develop the software are high on most process models. We found a clear majority of models and methodologies that take at least a partial understanding of customer requirements into account when developing the software. Only five of the process models and methodologies indicate that there is a need of a wellknown understanding and knowledge base of the requirements. Nearly all of our examined process models in the taxonomy, except two of them (WM and VA), construct and deliver prototypes to the customer in the development process of the software. With regard to the dimension "involvement of the customer", most of the process model studied involves customers from time to time in the development process (characteristic "regular"). The minority only includes them in the process at the beginning of the software development process. Five of the process models (XP, FDD, RUP, LSD, and MASAM) involve the customer throughout the development process in order to react to changes on in the requirements more rapidly.

Taking a more holistic view on our taxonomy in order to find and describe common characteristics of process models and methodologies, we saw that three process models have the same specifications. Mobile$\mathrm{D}, \mathrm{SM}$, and IPM follow iterative processes, have a high development effort, require partial knowledge of customer requirements, have incremental prototyping, and involve the customer regularly in the software development process.

\section{Discussion}

Our research contributes to theory is twofold. The taxonomy with its five dimensions serves as a starting point for situating and describing any research endeavor that is concerned with the phenomenon of mobile application development. With our conducted taxonomy we follow the recommendations for future 
research and outlook by Kemper \& Wolf [11] and expand the extant literature of software engineering and mobile application development by presenting a classification of existing processes and methodologies. The taxonomy developed here can serve as an advanced knowledge base for academics to conceptualize (iterative) process models on mobile application development, that more precisely meet the needs of mobile application developers [11].

In our second theoretical contribution, we showed that the taxonomy development approach of Nickerson et al. [12] is also applicable for process models and methodologies in general and in the specific mobile application-domain. We found that this procedure was appropriate to find relevant models and structure them into meaningful dimensions and characteristics. The subjective and objective endings conditions were suitable to lead our taxonomic process. In result, we expanded the knowledge in taxonomy building in a new research area, following a future research direction of Nickerson et al. [12].

From a practitioner's point of view, we connected our findings to domain-specific issues within the development for mobile applications. For example, process models can be classified by the dimension of the knowledge of the requirements of the customer at the beginning of the development process. In mobile application development, characteristics like the design of the user interface or security play a crucial role for the success $[48,49]$. User requirements can change rapidly [50]. Therefore, mobile application developers should follow an agile approach in software development. Novice developers of mobile applications can use this taxonomy as a starting point to receive an overview about existing process models and select the most appropriate methodology for their project. Experienced mobile developers in mobile development projects can compare the taxonomy with their own used methodologies and experiences and add new objects, characteristics and dimensions. The taxonomy can serve as a platform for communication and idea sharing for mobile application development.

Our taxonomy shows that the process models and methodologies, which we found in our second and fourth iteration can be characterized as nearly equal (except the MobiPDA approach). They all follow the iterative approach and have high development efforts. In addition, four of them postulate that the software developer only partially understand the needs of the customers. Due to the dynamics in the development of mobile applications (e.g., due to the degree of innovation of the new mobile application), the majority of the process models do not assume the full knowledge of the customers' software requirements at the beginning of the development process [11,51].
Each process model, which we found in the mobile application domain uses iterative prototyping within the development process. According to Keil \& Carmel [42], prototyping, in general, plays a crucial role in software development procedures and is also adapted into mobile application development processes. Differences can be derived in the dimension of "involvement of the customer". While Mobile-D and IPM regularly involve customers in the process from time to time, LSD and MASAM permanently involve customers in the development process. For software development processes, it is empirically proven that a strong communication and participation between developers and customers, leads to more satisfying end-user software [42]. Not in the development phase, but also in the post-development phase of the mobile application, there are positive effects in the satisfaction through a communication of developers and end users [52]. This also can be discussed in terms of mobile application specific development issues. Higher user involvement in software projects is recommended for those projects where the user acceptance is important and the development process is unstructured [53]. This seems especially true for mobile application development projects.

An exception of the specific process model used for mobile application development is the MobiPDA approach. It is, compared to the other models described before, classified with low development efforts. The customer gives the information regarding the requirements only at the beginning to the software development team. In addition, customer's requirements for the mobile application must be wellknown. Like in the other process models in the second and the fourth iteration, prototyping happens incremental. MobiPDA has a more explicit problem definition and idea development stage [47]. Due to these extensive communication phases with the customer at the beginning of the mobile application development, the requirements of the customers are well-known to the developers in the current process.

Our discussion of the taxonomy and the characteristics, such as the iterative approach of our found mobile application process models, showed that most of them have similar appearances. The findings of our research indicate that iterative or agile process models and methodologies are more suitable for mobile application development. Because of domainspecific issues, such as rapid change in customer requirements in mobile application development, agile methods are more flexible on changes in the development process [54]. These findings are consistent with other researchers $[1,48]$. 


\section{Limitations and future research}

We will now outline the limitations of our study and suggest some future research directions.

The first limitation is the inability of our taxonomy to affirm completeness in case of all process models and methodologies. We examined the literature and discontinued the research process after all objective and subjective ending conditions were met. As mentioned by Nickerson et al. [12], the definition of the meta-characteristics or ending conditions is part of a subjective procedure of the authors. For example, in our research, we found a process model for mobile application development, called Mobia modeler. The prototyping dimension at Mobia modelers procedure is not clearly defined, the classification in both characteristics could be possible [47,55]. Because of this unclear issue, we did not include Mobia modeler into our taxonomy. As Nakatsu et al. [56] mentioned for taxonomic approaches, there is always a critical tradeoff between over-simplicity and comprehensiveness. But because of the extendable nature of our taxonomy, we think that future research and practitioners could expand this taxonomy by own knowledge or real examples of development as well as by adding, changing, deleting, or combining dimensions. In this context, interviews with leading organizations would also provide a suitable mechanism for establishing what was essential and unique about mobile application development from the perspective of practice. The results of our study as well as the results of this future qualitative study can lead to an extended taxonomy by combination, synthesis, and coding. In addition, a validation and evaluation of our findings is necessary in order to check its usefulness and to tests its efficacy by categorizing more mechanisms. This could lead to additional dimensions and configurations and therefore new knowledge.

Second, we focus in our examination on the taxonomy development of process models and methodologies in software engineering. There is already a large amount of literature for the established software engineering process models, such as SCRUM or the SM available. Therefore, we try to focus more on domain-specific argumentations for process models and their execution inside the mobile application area. From our viewpoint, the literature about this special issue is restricted. Future research directions should analyze this domain-specific problems or key characteristics in more detail [8]. This would help all involved people in the mobile application development process to receive new insights of knowledge and crucial factors in this field.
Another limitation is the inflexibility of classical process models in a company environment [24,57]. Unexpected events or shifting circumstances can lead to a change in the originally planned process [58]. This seems especially true for mobile application development processes [11]. As stated in our discussion, agile software development methods are appropriate for mobile application development. A possible continuative avenue for future research would be a flexible design theory (e.g., $[33,59])$ constructed under the design science research paradigm [31], which incorporates mobile application specific requirements for software development. On the one hand, this meta-artifact (e.g., orientated on the format of the Software \& Systems Process Engineering MetaModel (SPEM)) [60] could take into account the knowledge of software development in general and, on the other hand, specific success factors and development issues regarding the design (e.g., about the UI) of various mobile applications, outlined in the theoretical background of our study.

\section{Conclusion}

The development of mobile applications and the adequate fulfillment of customer requirements play an ongoing important role in practice. We observed that the academic literature about experiences and applications in using process models in the specific mobile application development domain is straightforward. First, we gave a theoretical background of characteristics and domain-specific software development issues in mobile applications. Furthermore, a knowledge background of process models and methodologies in software engineering and mobile application development was given. We described our methodology of taxonomy development in section three. We contribute to existing literature in the field of mobile application development by building and presenting a taxonomy for process models and methodologies in this domain. We structured them into several dimensions and characteristics and conclude to specific issues related to mobile application development. We showed that the used taxonomic approach is also applicable inside the mobile application development domain. From the perspective from a practitioner, we gave several decision guidance to select the most accurate process model for a specific dimension of the mobile application. Novice and experienced software developers will be helped in their growing task to fulfil customer's specific requirements for mobile applications. In addition, we outlined a number of limitations of our research and implied research 
directions for software development into the domain of mobile applications. We hope that our research has structured the community's knowledge of process models and methodologies and has extended its application to the growing area of mobile application development.

\section{References}

[1] A. I. Wasserman, "Software engineering issues for mobile application development", in Proc. of the FSE/SDP workshop on future of software engineering research, New York, NY, USA: ACM, 2010, pp. 397-400.

[2] Statista, "Number of apps available in leading app stores", 2018 , https://www.statista.com/statistics/276623/number-of-appsavailable-in-leading-app-stores/.

[3] M. Salo and M. Makkonen, "Why do users switch mobile applications? Trialing behavior as a predecessor of switching behavior", Communications of the Association for Information Systems, vol. 42, no. 1, 2018, pp. 386-407.

[4] H. Hoehle and V. Venkatesh, "Mobile application usability: Conceptualization and instrument development", MIS Quarterly, vol. 39, no. 2, 2015, pp. 435-472.

[5] A. Charland and B. Leroux, "Mobile application development: Web vs. native", Communications of the Association for Information Systems, vol. 54, no. 5, 2011, pp. 49-53.

[6] Y. Choi, J.-S. Yang, and J. Jeong, “Application framework for multi-platform mobile application software development", in Proc. of the 11 th International Conference on Advanced Communication Technology, 2009, pp. 208213.

[7] T. A. Majchrzak, A. Biørn-Hansen, and T.-M. Grønli, "Progressive web apps: The definite approach to crossplatform development?", in Proc. of the 51st Hawaii International Conference on System Sciences (HICCS), 2018, pp. 5735-5744.

[8] H. K. Flora, X. Wang, and S. V. Chande, "An investigation on the characteristics of mobile applications: A Survey Study", I.J. Information Technology and Computer Science, vol. 11, 2014, pp. 21-27.

[9] N. Singh and D. Soni, "Proposing new model for effort estimation of mobile application development", International Journal of Computer Applications, vol. 170, no. 3, 2017, pp. 14-18.

[10] T .A. Majchrzak and T.-M. Grønli, "Introduction to the HICSS-51 Software Development for Mobile Devices, Wearables, and the Internet-of-Things Minitrack", in Proc. of the 51st Hawaii International Conference on System Sciences (HICCS), 2018, p. 5686.

[11] H. G. Kemper and E. Wolf, "Iterative process models for mobile application systems: A framework", in Proc. of the 23th International Conference on Information Systems (ICIS), 2002, pp. 401-413.

[12] R. C. Nickerson, U. Varshney, and J. Muntermann, "A method for taxonomy development and its application in information systems", European Journal of Information Systems, vol. 22, 2013, pp. 336-359.
[13] H. Barki, S. Rivard, and J. Talbot, "An information systems keyword classification scheme". MIS Quarterly, vol. 12, no. 2, 1988, pp. 299-322.

[14] R. L. Glass and I. Vessey, "Contemporary applicationdomain taxonomies", IEEE Software, vol. 4, no. 12, 1995, pp. 6378.

[15] D. H. McKnight and N. L. Chervany, "What trust means in e-commerce customer relationships: An interdisciplinary conceptual typology", International Journal of Electronic Commerce, vol. 6, no. 2, 2001, pp. 35-59.

[16] S. P. Han, S. Park, S., and W. Oh, "Mobile app analytics: A multiple discrete-continuous choice framework", MIS Quarterly, vol. 40, no. 4, 2016, pp. 9831008.

[17] J. Dehlinger and J. Dixon, "Mobile application software engineering: Challenges and research directions", in Proc. of the Workshop on Mobile Software Engineering, 2011, pp. 29-32.

[18] M. E. Joorabchi, A. Mesbah, and P. Kruchten, "Real Challenges in Mobile App Development", in Proc. of the International Symposium on Empirical Software Engineering and Measurement, 2013, pp. 15-24.

[19] S. S. Oyelere, J. Suhonen, G. M. Wajiga, and E. Sutinen, "Design, development, and evaluation of a mobile learning application for computing education", Education and Information Technologies, vol. 23, no. 1, 2018, pp. 467495.

[20] Pressman, R. S., and Maxim, B. R., Software engineering: A practitioner's approach, McGraw-Hill, 5 edition, 2015.

[21] A. Langley, "Strategies for theorizing from process data", Academy of Management Review, vol. 24, no. 4, 1999, pp. 691-710.

[22] N. Kock, J. Verville, A. Danesh-Pajou, and D. DeLuca, "Communication flow orientation in business process modeling and its effect on redesign success: Results from a field study", Decision Support Systems, vol. 46, 2009, pp. 562-575.

[23] Dumas M., La Rosa M., Mendling J., and Reijers H.A., Fundamentals of business process management, Springer, New York, 2013.

[24] D. Simões, P. Antunes, and L. Carriço, "Eliciting and modeling business process stories", Business \& Information Systems Engineering, vol. 60, no. 2, 2018, pp. 115-132.

[25] Scacchi, W., Process models in software engineering, Encyclopedia of software engineering, Wiley \& Sons, Hoboken, 2002, pp. 1-24.

[26] B. Boehm, "A spiral model of software development and enhancement", Computer, vol. 20, no. 9, 1987, pp. 6172.

[27] C. Rieger, "Evaluating a graphical model-driven approach to codeless business app development", in Proc. of the 51st Hawaii International Conference on System Sciences (HICCS), 2018, pp. 5725-5734.

[28] G. Remane, R. C. Nickerson, A. Hanelt, J. F. Tesch, and L. Kolbe, "A taxonomy of carsharing business models", in Proc. of the 37th International Conference on Information Systems (ICIS), 2016, pp. 1-19.

[29] V. Mrass, L. Manhei Manhai, and C. Peters, "Towards a taxonomy of digital work", in Proc. of the 25th European 
Conference on Information Systems (ECIS), 2017, pp. 25152524.

[30] N. Prat, I. Comyn-Wattiau, and J. Akoka, "A taxonomy of evaluation methods for information systems artifacts", Journal of Management Information Systems, vol. 32, no. 3, 2015, pp. 229-267.

[31] A. R. Hevner, S. T. March, J. Park, and S. Ram, "Design science in information systems research", MIS Quarterly, vol. 28, no. 1, 2004, pp. 75-105.

[32] H. Gimpel, D. Rau, and M. Röglinger, "Understanding FinTech start-ups - a taxonomy of consumer-oriented service offerings", Electronic Markets, vol. 22, 2017, pp. 120.

[33] J. Iversen, I. Mathiassen, and P. Nielsen, "Managing process risk in software process improvement: An action research approach", MIS Quarterly, vol. 28, no. 3, 2004, pp. 395-434.

[34] W. W. Royce, "Managing the development of large software systems", in Proc. of the 9th International Conference on Software Engineering, 1970, pp. 328-338.

[35] C. Bucanac, The V-Model, 1999 www.bucanac.com/documents/The_V-Model.pdf.

[36] McDermid, J., and Rook, P., Software Engineer's Reference Book, CRC Press, Boca Raton, 1993.

[37] Beck, K., Extreme Programming Explained: Embrace Change, Addison-Wesley, Boston, 2004.

[38] Schwaber, K., and Beedle, M., Agile Software Development with SCRUM, Prentice Hall, Upper Saddle River, 2001.

[39] Stapleton, J., DSDM, dynamic systems development method: the method in practice, University Press, Cambridge, 1997.

[40] Palmer, S. R., and Felsing, M., A practical guide to feature-driven development, Pearson Education, New York, 2001.

[41] Kruchten, P. (2004). The rational unified process: an introduction, Addison-Wesley Professional, Boston, 2004.

[42] M. Keil and E. Carmel, "Customer-developer links in software development", Communications of the Association for Information Systems, vol. 38, no. 5, 1995) pp. 33-44.

[43] P. Abrahamsson, A. Hanhineva, H. Hulkko, T. Ihme, J. Jäälinoja, M. Korkala, J. Koskela, P. Kyllönen, and O. Salo, "Mobile-D: An agile approach for mobile application development", in Comp. to the 19th annual ACM SIGPLAN conference on object-oriented programming systems, languages, and applications, 2004, pp. 174-175.

[44] Poppendieck, M., and Poppendieck, T., Lean software development: An agile toolkit, Addison-Wesley, Boston, 2003.

[45] J. Webster and R. T. Watson, "Analyzing the past to prepare for the future: Writing a literature review", MIS Quarterly, vol. 26, no. 2, 2002. pp. xiii-xxiii.

[46] Y.-J. Jeong, J.-H. Lee, and G.-S. Shin, "Development process of mobile application SW based on agile methodology", in Proc. of the 10th International Conference on Advanced Communication Technology (ICAT), 2008, pp. 362-366.

[47] K. N. Tran, and H. Q. Nguyen, "MobiPDA: A systematic approach to mobile-application development", in Proc. of the International Conference on Computational Science and its Applications, 2013, pp. 232-247.
[48] C. Scharff and R. Verma, "Scrum to support mobile application development projects in a just-in-time learning context", in Proc. of the 2010 ICSE Workshop on Cooperative and Human Aspects of Software Engineering, 2010, pp. 25-31.

[49] M. Popa, "Considerations regarding the cross-platform mobile application development process", Economy Informatics, vol. 13, no. 1, 2013, pp. 40-52.

[50] A. Ashishdeep, J. Bhatia, and K. Varma, "Software process models for mobile application development", International Journal of Computer Science \& Communication, vol. 7, no. 1, 2015, pp.150-153.

[51] V. N. Inukollu, D. D. Keshamoni, T. Kang, and M. Inukollu, "Factors influencing quality of mobile apps: Role of mobile app development life cycle", International Journal of Software Engineering \& Application, vol. 5, no. 5, 2014, pp. 15-34.

[52] S. McIlroy, W. Shang, N. Ali, and A. E. Hassan, "Is it worth responding to reviews? Studying the top free apps in Google Play", IEEE Software, vol. 34, no. 3, 2017, pp. 6471.

[53] B. Ives, B. and M. H. Olson, "User involvement and MIS success: A review of research", Management Science, vol. 30, no. 5, 1984, pp. 586-603.

[54] S. B. Kaleel and S. Harishankar, "Applying agile methodology in mobile software engineering: Android application development and its challenges", Computer Science Technical Reports, 2013.

[55] F. Balagtas-Fernandez, M. Tafelmayer, and $\mathrm{H}$. Hussmann, "Mobia modeler: Easing the creation process of mobile applications for non-technical users", in Proc. of the 15th International Conference on Intelligent User Interfaces, 2010, pp. 269-272.

[56] R. T. Nakatsu, E. B. Grossman, and C. L. Iacovou, "A taxonomy of crowdsourcing based on task complexity", Journal of Information Science, vol. 40, no. 6, 2014, pp. 823834.

[57] A. Lindsay, D. Downs, and K. Lunn, "Business processes-attempts to find a definition", Information Software Technology, vol. 45, 2013, pp. 1015-1019.

[58] L. Jonnavithula, P. Antunes, J. Cranefield, and J. A. Pino, "Organisational issues in modelling business processes: An activity-based inventory and directions for research", in Proc. of the 19th Pacific Asia Conference on Information Systems (PACIS), 2015, pp. 1-16.

[59] S. Gregor and D. Jones, "The anatomy of a design theory", Journal of the Association of Information Systems, vol. 8, 2007, pp. 312-335.

[60] Object Management Group, Software \& systems process engineering meta-model specification, 2008, https://www.omg.org/spec/SPEM/2.0. 